\title{
Fission fragment yields and total kinetic energy release in neutron-induced fission of ${ }^{235,238} \mathrm{U}$, and ${ }^{239} \mathrm{Pu}$
}

\author{
F. Tovesson, D. Duke, V. Geppert-Kleinrath, B. Manning, D. Mayorov, S. Mosby, and K. Schmitt \\ Los Alamos National Laboratory, Los Alamos, New Mexico 77843, USA
}

\begin{abstract}
Different aspects of the nuclear fission process have been studied at Los Alamos Neutron Science Center (LANSCE) using various instruments and experimental techniques. Properties of the fragments emitted in fission have been investigated using Frisch-grid ionization chambers, a Time Projection Chamber (TPC), and the SPIDER instrument which employs the $2 \mathrm{v}-2 \mathrm{E}$ method. These instruments and experimental techniques have been used to determine fission product mass yields, the energy dependent total kinetic energy (TKE) release, and anisotropy in neutron-induced fission of U-235, $\mathrm{U}-238$ and $\mathrm{Pu}-239$.
\end{abstract}

\section{Introduction}

Fission nuclear data is important for, and impacts the performance of, several nuclear technologies. Furthermore, the fission process is highly complex and is still, more than 75 years after its discovery, not completely understood. New measurements, particularly of correlated observables, allows us to develop more sophisticated theoretical models and to eventually have truly predictive capabilities for the physics of fission. The technologies that are impacted cover those deployed in service of the nuclear energy, defense, non-proliferation, and nuclear forensics efforts. An experimental program at Los Alamos National Laboratory has been developed to measure several aspects of actinide fission, including product yields and kinetic energy release. These quantities were obtained using different instruments, each designed to capture a measurement of a specific fission observable with high-resolution or with high-statistics. Often, correlations between relevant observables were determined during the measurements. This paper will describe the experiments carried out on U-235, $\mathrm{U}-238$ and $\mathrm{Pu}-239$.

\section{Experimental techniques}

The experiments were performed at the Los Alamos Neutron Science Center (LANSCE) at Los Alamos National Laboratory. The $800-\mathrm{MeV}$ proton accelerator at LANSCE is used to deliver a pulsed beam to a number of facilities, including the Weapons Neutron Research (WNR) and Lujan Center where multiple flightpaths or instrumental halls are positioned about the source of the neutron beam.

At WNR the primary proton beam hits a water-cooled tungsten target which creates fast neutrons through the spallation process. In the present experiments, we used a 10-m flightpath located at 90 degrees relative to the proton beam at WNR. This is a short flight path, which results in a high 
neutron flux, and the 90-degree spallation has an average neutron energy of about $2 \mathrm{MeV}$, which is appropriate for fast neutron-induced fission research. The neutron time-of-flight (TOF) method was used to determine the incident neutron energies.

The Lujan Center also takes advantage of spallation on tungsten for neutron production, but here the target is surrounded by moderating material, resulting in highly thermalized neutron spectra on the various flightpaths. In the current experiments, flightpath 12 was used, which has a cold moderator. Again, the neutron TOF method was used to determine the incident neutron energies.

The different detectors used for fission studies were chosen to be complimentary in the information that they provide. The SPIDER $2 \mathrm{v}-2 \mathrm{E}$ spectrometer provides high-accuracy measurements of fission product mass, but with relatively low efficiency. The ionization chambers provide lower mass resolution, but are highly efficient and have high energy resolution. The time projection chamber (TPC) has high detection efficiency, somewhat lower energy resolution than the ionization chambers, but provides highly accurate particle tracking information.

\subsection{Ionization chambers}

Frisch-grid ionization chambers were used to study the total kinetic energy release in fission, the gross distributions of fission product masses, and the correlation between the two at difference incident neutron energies.

The ionization chambers and how they are operated are described in reference [1]. The chambers measure the kinetic energy of both of the fragments emitted in binary fission. The sum of the energies is the total kinetic energy release (TKE) of the fragments. Furthermore, the energies of the individual fragments can be used to infer the masses, by using momentum and mass conservation. This is known as the 2E-method, and it relies on previous knowledge of the average number of neutrons emitted for a given fragment mass. The correction limits the mass resolution to 4-5 atomic mass units (amu).

\subsection{Spectrometer for ion determination in fission research (SPIDER)}

The SPIDER instrument (Fig. 1) measures the velocity and kinetic energy release in the two fragments emitted in binary fission. This allows the fission product masses to be obtained with 1-2 amu resolution, as demonstrated at the ILL in the 1980's. The prototype version of the SPIDER instrument was built with two detector arms, with at total solid angle coverage of about $0.05 \%$. The next version of the instrument is currently being constructed and will have 16 detector arms, bringing the total angular efficiency close to $1 \%$.

\subsection{Time projection chamber (TPC)}

The Time Projection Chamber (TPC) is described in detail in reference [2]. The TPC was developed mainly for high precision fission cross section measurements, but can also be used to study other aspects of the nuclear fission process. The detector provides full, 3D particle tracking, and is therefore ideal for measuring anisotropy and angular distributions of fission fragments. The tracking is achieved in the TPC with a pixilated readout that provides the (x,y)-projection of a given particle's track, which then is combined with drift time measurements to reconstruct the $3 \mathrm{D}$ tracks.

\section{Results}

\subsection{Total kinetic energy release (TKE)}

The total kinetic energy release in fission demonstrates a general trend of decreasing with increasing incident neutron energy [3]. There is however, only limited experimental information for the major 


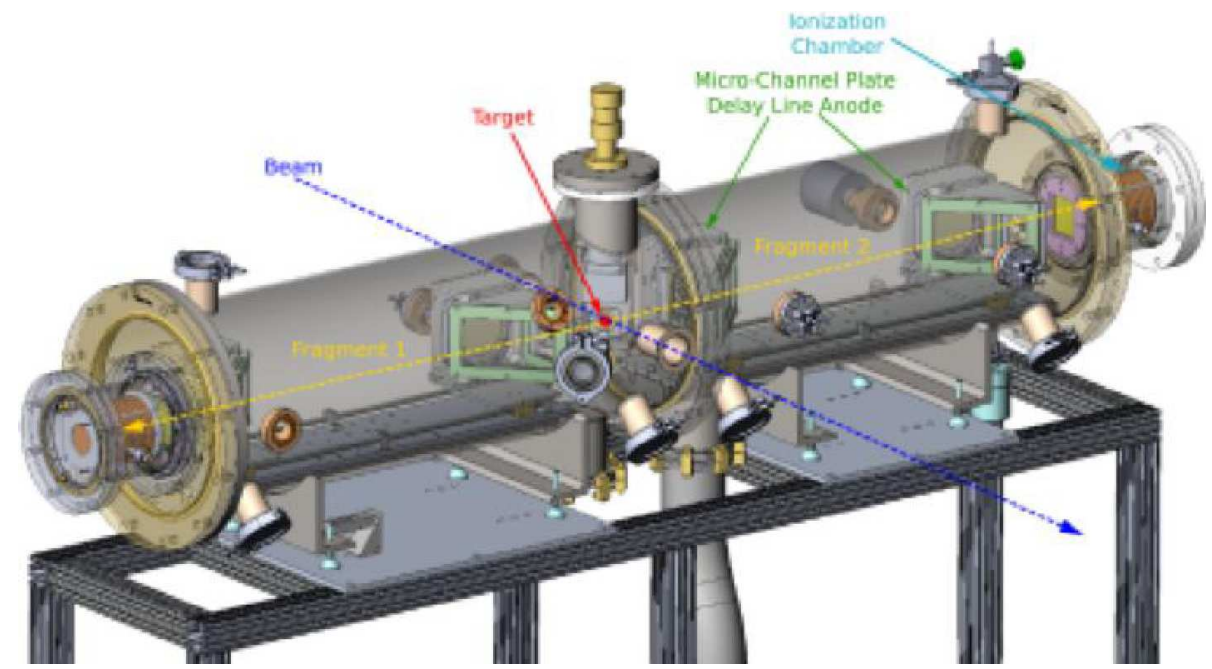

Figure 1. Schematic view of the SPIDER instrument.

actinides above 5-10 MeV. Furthermore, some of the fine structures are not well measured, such as the sudden fluctuations in TKE at the thresholds for multi-chance fission channels.

The TKE as a function of incident neutron energy for U-238 [4], U-235, and Pu-239 [5] is shown in Fig. 2. These data sets extend out to $30 \mathrm{MeV}$, which helps constrain the TKE in the region around $14 \mathrm{MeV}$. This is of particular interest to applications aspects of nuclear technology. For all systems, the same trends around the opening of multi-channel fission is observed where the TKE increases locally, only to decrease again at higher neutron energies.

\subsection{Mass yields}

The mass yields for thermal neutron-induced fission were measured with SPIDER for U-235 and Pu239, and the preliminary result for the former is shown in Fig. 3. Good agreement with the ENDF evaluation is generally observed, although some differences for individual yields are evident. For U235 there is high confidence in the evaluation, and the difference can likely be attribute to experimental resolution effects and potentially calibration issues. For $\mathrm{Pu}-239$ there is less previous experimental data, and the evaluations are somewhat less reliable.

The Frisch-grid ionization chamber was used to study the change in mass yields as a function of incident neutron energy. The result for U-238 is shown in Fig. 4. While the mass resolution is lower than for the SPIDER instrument, the general trends of the mass yield distributions are clearly observed. As the incident neutron energy increases the asymmetric distribution at low energies changes and a symmetric component is more and more evident. This is a well-known effect, but there is again very limited experimental information above $5-10 \mathrm{MeV}$.

\subsection{Fragment anisotropy}

The fission fragment anisotropy in U-235 was measured with the Time Projection Chamber. The results generally agree with literature data, and a manuscript describing the results has been submitted 

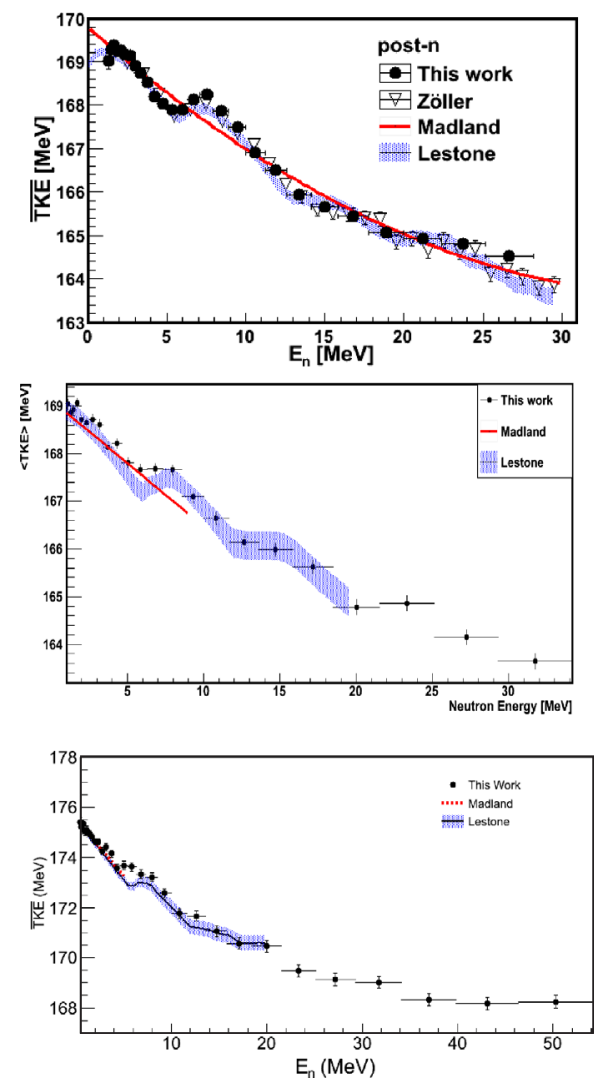

Figure 2. Total kinetic energy (TKE) for U-238 (top), U-235 (middle), and Pu-239 (bottom).

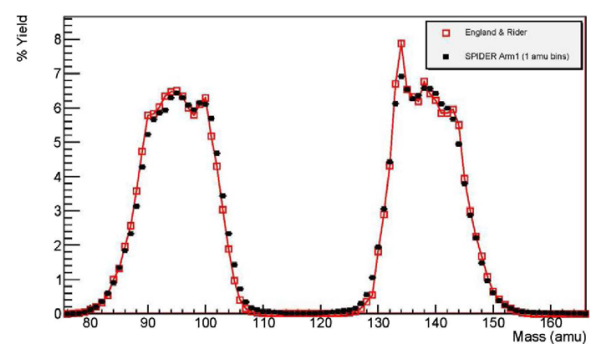

Figure 3. Preliminary mass yields for thermal neutron-induced fission of U-235.

for publication. The TPC is capable of measuring the full angular distributions, but for the initial work the focus was given to the anisotropy, since there is some previous work available for a comparison. 


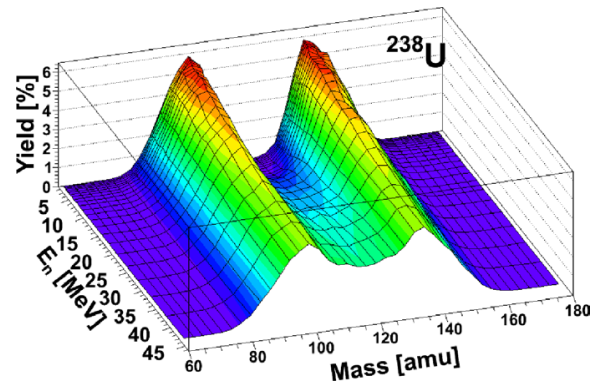

Figure 4. Mass yields of U-235 measured up to $45 \mathrm{MeV}$ of incident neutron energy.

\section{Conclusions and outlook}

The work presented here shows the current capabilities of studying fission fragment properties at LANSCE. The results provide rather comprehensive information about fission fragments over a wide range of neutron energies for the major actinides. This information can help in benchmarking and guiding advanced fission models, as well as in providing nuclear data relevant to nuclear technology.

One of the missing capabilities for the fission fragment studies is the ability to measure mass yields with high resolution not only for thermal neutrons, but also in the fast region. This is driving the development of MegaSPIDER, which is currently being assembled. This new instrument will be used for experiments at WNR to extend the previous measurements with thermal neutrons to the fast range.

Another further extension of this program is to study the minor actinides. This is already in progress, with a study of U-233. In addition, there are plans for Np-237 and other systems relevant for nuclear reactor fuel cycles such as $\mathrm{Pu}-240$ and $\mathrm{Pu}-242$.

\section{References}

[1] S. Mosby, F. Tovesson, A. Couture, D. Duke, V. Kleinrath, R. Meharchand, K. Meierbachtol, J.M. O’Donnell, B. Perdue, D. Richman, D. Shields, Nucl. Instr. and Meth. A 757, 75 (2014)

[2] M. Heffner, D.M. Asner, R.G. Baker, et al., Nucl. Instr. and Meth. A 759, 50 (2014)

[3] D.G. Madland, Nucl. Phys. A 772, 113 (2006)

[4] D.L. Duke, F. Tovesson, A.B. Laptev, S. Mosby, F.-J. Hambsch, T. Brys, and M. Vidali, Phys. Rev. C 94, 054604 (2016)

[5] K. Meierbachtol, F. Tovesson, D.L. Duke, V. Kleinrath, B. Manning, R. Meharchand, S. Mosby, and D. Shields, Phys. Rev. C 94, 034611 (2016) 
